\title{
En memoria: nota necrológica Billie Jean Isbell 1937-2021
}

\section{Catherine Allen}

(iD) https://orcid.org/0000-0002-4715-0108.

Profesora emérita, Universidad George Washington, Estados Unidos

kitallen@gwu.edu

Billie Jean Richerson Isbell, profesora emérita de antropología en la Universidad de Cornell, falleció el 26 de junio de 2021 en Santa Fe, Nuevo México, por complicaciones derivadas de una caída. Fue una etnógrafa consumada y se distinguió en estudios andinos. Su libro To Defend Ourselves: Ecology and Ritual in an Andean Village (1978) — un estudio de la vida ritual, parentesco, organización sociopolítica, migración urbana y la política en la comunidad de Chuschi, Perú - se ha convertido en un clásico, publicado varias veces y traducido al español: Para defendernos: ecología y ritual en un pueblo andino (Centro de Estudios Rurales Bartolomé de las Casas,2007).

Billie Jean Richerson nació en Lark, Utah, un pueblo de obreros mineros de carbón, y creció en San Antonio, Texas. De niña sufrió de fiebre reumática, y la consiguiente artritis reumatoide la afectó periódicamente durante toda su vida. A los dieciocho años se fue de casa, primero a Jackson Hole (Wyoming) y, posteriormente, a San Francisco (California), donde obtuvo el título de profesora de natación y trabajó en la Asociación de Mujeres de la YWCA en varios puestos. En 1960 ingresó en la Universidad Estatal de San Francisco, con la idea de seguir una carrera en fisioterapeuta, pero se sintió inspirada por la antropología. Billie Jean fue la primera de su familia en graduarse en una universidad, un logro importante si se tiene en cuenta que su madre le pidió que abandonara la secundaria en su último año para que buscara un trabajo y ayudara a la familia. Eso fue a la muerte de su padre, un minero que murió de silicosis.

Billie Jean se ganaba el pan como ama de llaves, niñera, mecanógrafa e instructora de seguridad para salvavidas. En 1962 se casó con William (Bill) Isbell, y al año siguiente, ella y su marido se enrolaron en el Cuerpo de Paz en 
Colombia, primero un año en el pueblo de Tunja, Boyacá, y otro en Medellín, Antioquia. Durante ese tiempo, también dio a luz a su hija, Diana Richerson Isbell.

En 1965, los Isbell, al terminar su servicio en el Cuerpo de Paz, regresaron a los Estados Unidos y se matricularon en la Universidad de Illinois UrbanaChampaign, donde Billie Jean completó los créditos finales de su licenciatura y continuó como estudiante de posgrado de antropología. En 1967-68 participó en el proyecto Río Pampas, un estudio etnográfico y etnohistórico de un grupo de pueblos en la región del río Pampas de Ayacucho, Perú, que estaban bastante aislados, nunca incorporados a haciendas, y de quienes existían buenos registros históricos. El proyecto fue creado y dirigido por R. Tom Zuidema, quien se convirtió en el asesor académico y mentor intelectual de Billie Jean. Los Isbell trabajaron en el pueblo quechua de Chuschi, acompañados por su hija pequeña y por la madre de Billie Jean. Al ser una familia de tres generaciones, ellos encajaron fácilmente en el ambiente social del pueblo andino, factor que permitió a Billie Jean tener éxito en sus investigaciones etnográficas. Vivió en Chuschi entre enero y agosto de 1968. Animada por Zuidema, decidió llevar a cabo una etnografía general del pueblo chuschino para su proyecto de doctorado. Después de un verano de formación en quechua en Cochabamba, Bolivia, regresó para otro periodo prolongado en el pueblo en 1969-1970.

Billie Jean continuó su investigación posdoctoral en Chuschi en 1974-1975, como becaria del NIMH (Instituto Nacional de Salud Mental de los Estados Unidos). Sobre la base de estos periodos de trabajo de campo, completó el manuscrito de To Defend Ourselves. Para entonces, ya enseñaba antropología en la Universidad Estatal de Nueva York en Albany — de 1972 a 1975 — y se estaba convirtiendo en una mentora muy popular entre los estudiantes, especialmente entre las alumnas. En 1976 se incorporó a la facultad de Antropología de la Universidad de Cornell y fue la primera mujer contratada como profesora principal en ese departamento. Fue directora del Programa Andino del Instituto Internacional de Alimentación, Agricultura y Desarrollo de Cornell (1990-2002) y del Programa de Estudios Latinoamericanos de Cornell (1987-1993, 2001-2002). A lo largo de los años organizó muchas conferencias memorables y eventos especiales sobre temas que iban desde los derechos humanos hasta la estética intercultural. Se jubiló en 2002, en parte debido a su delicada salud.

Aunque era principalmente etnógrafa, los intereses de Billie Jean incluían la etnohistoria y la arqueología. En 1981-1982, colaboró con Zuidema y el astrónomo Anthony Aveni, fotografiando y cartografiando alineaciones astronómicas en sitios incas del Cusco, centrándose especialmente en las observaciones del paso del 
sol por el cenit, tal como se refleja en la arquitectura inca. Ella y Aveni viajaron por la costa norte de Perú visitando varios sitios, y Billie Jean continuó hacia Ecuador y Bolivia para seguir observando cómo cambiaban las alineaciones en diferentes latitudes. El artículo resultante se publicó en un volumen sobre etno y arqueoastronomía en los trópicos, editado por Aveni y Urton, y está disponible en Cornell University eCommons https:/lecommons.cornell.edu/handle/1813/2193. Pensó en continuar esta investigación con un proyecto llamado «La sombra del tiempo», pero la urgencia de la violencia que vivió el Perú tuvo prioridad y desvió su atención durante los siguientes veinticinco años a otros temas.

En 1985, recibió una carta de un compadre de Chuschi en la que este le describía cómo el pueblo había sido incendiado y casi totalmente abandonado. Profundamente afectada por esta y otras devastaciones que sucedieron durante la insurgencia de Sendero Luminoso, Billie Jean obtuvo una beca del Programa de Estudios para la Paz de Cornell y viajó a Lima para ponerse en contacto con organizaciones de derechos humanos y asociaciones de víctimas. Durante las dos décadas siguientes trabajó intensamente con estos grupos para grabar los testimonios de los refugiados en Lima. En 1993 recorrió las universidades en los Estados Unidos, durante exhibió una arpillera que testimoniaba a los desaparecidos, cosida por los familiares de las víctimas. Desilusionada con el discurso de las ciencias sociales como medio para expresar estas emotivas vivencias, comenzó a experimentar con la manera de representar adecuadamente las experiencias sobre la violencia en esos tiempos. Cuando, en 1985, Waveland Press publicó una nueva edición de To Defend Ourselves, Billie Jean incluyó un nuevo capítulo sobre los acontecimientos que se estaban desarrollando en Chuschi, así como un prefacio en el que describía críticamente que su enfoque estructuralista la había distanciado de las realidades políticas que se desarrollaban en esa región. Pasó a nuevos enfoques, esforzándose por transmitir la realidad de la guerra a través otros modos de composición incorporando testimonios y diálogos (por ejemplo, «Violence in Peru: Performances and Dialogues», en The American Anthropologist, 1998, recuperado de https://ecommons.cornell.edu/handle/1813/2195). En 2009 se publicó Finding Cholita, una obra de ficción que a su vez tendría aspectos que exploran los efectos a largo plazo de la violencia crónica en Ayacucho. Este libro recibió una mención honorífica del premio Victor Turner.

Los aspectos de la identidad y género en las culturas andinas fue otro interés permanente en sus trabajos; el tema recorre como un leitmotiv gran parte de la investigación y los escritos de Billie Jean. Su artículo «De inmaduro a duro» (publicado en 1997 y recuperado de https://ecommons.cornell.edu/handle/1813/2474) 
se basa en fuentes etnográficas y etnohistóricas para argumentar que el género andino pasa por una serie de transformaciones a lo largo del ciclo vital de un individuo, una importante idea que dio una nueva complejidad a las teorías de la complementariedad de género en los Andes. El enfoque de género adquirió una nueva dimensión a partir del año 2000, cuando Diana hizo la transición a hombre y se convirtió en su hijo, Cid. Billie Jean redactó una novela sobre la familia de un niño transgénero pero, por motivos de salud, no llegó a completarla.

En el último trimestre de 2002, Billie Jean se enteró de que la comunidad indígena de Vicos, en Ancash, había manifestado su interés por volver a conectarse con el «señor Cornell». Así era como los ancianos de la comunidad se referían al proyecto Perú-Cornell, un renombrado programa de antropología aplicada dirigido por Cornell y antropólogos peruanos entre 1952 y finales de la década de 1960. Una vez que se enteró de esta petición, Billie Jean movilizó el apoyo del Programa de Estudios Latinoamericanos y trabajó durante los cinco años siguientes con Florencia Zapata y Jorge Recharte, del Instituto de Montaña en toda una serie de iniciativas, algunas de las cuales se describen en https://vicosperu.cornell.edu/ vicos-site/. Gracias a su apoyo y orientación, una delegación de Vicos visitó el campus de Cornell y se llevaron a su comunidad una colección de artefactos de Vicos y cientos de fotografías, horas de película y mapas que se encontraban en la biblioteca de la universidad. Estos materiales ahora se exponen en La Casa de los Abuelos, un pequeño museo gestionado por la comunidad y dedicado a la cultura vicosina. Los jóvenes de Vicos utilizaron los materiales para una actividad de memoria social para reflexionar, junto con sus mayores, sobre la historia del cambio social y ecológico en su comunidad, incluidos los cambios creados por el Proyecto Perú-Cornell.

El resultado fue un libro escrito por la comunidad, Memorias de la Comunidad de Vicos: Así recordamos con alegría (Florencia Zapata; Comunidad Campesina de Vicos (Peru); Instituto de Montaña. Huaraz, Perú: Corporación Gráfica Andina, 2005). Miembros de la Universidad de Cornell, incluyendo a Billie Jean, fueron recibidos en la comunidad para ese evento. Esta historia ilustra cómo Billie Jean era una buena articuladora de relaciones humanas.

La última publicación de Billie Jean fue un capítulo en Return to the Village: Community Ethnographies and the Study of Andean Culture in Retrospective (F. Ferreira, ed. Londres: University of London Press; acceso abierto: http://www. humanities-digital-library.org), titulado «Reflections on Fieldwork in Chuschi». Este ensayo relata su evolución como antropóloga, escritora y portavoz de los derechos humanos. En su párrafo final comenta: «Encontré refugio en el arte y 
la ficción cuando no pude encontrar una voz, una metodología o un paradigma para lo que estaba experimentando como antropóloga trabajando en una zona de los Andes desgarrada por la guerra. Encontré esa voz en la narrativa y en contar las historias de la gente».

Billie Jean poseía una enorme joie de vivre que le permitió superar la pobreza de la infancia y la mala salud de toda la vida con gracia y buen humor. Vivía con estilo, generosidad y un gran sentido de la diversión. Sus amigos recordarán durante mucho tiempo su espectacular jardín, su deliciosa cocina y las tardes que pasaban conversando en su casa cerca de Ithaca.

Su matrimonio con William Isbell terminó en divorcio después de veinticuatro años. La sobreviven su hijo y su nuera, Cid y Medina Isbell, su exmarido Bill y muchos amigos de todo el mundo. La colección Andes de Billie Jean Isbell, que contiene unas 1500 fotos comentadas, canciones y publicaciones seleccionadas de su vida está disponible en http://isbellandes.library.cornell.edu/, como proyecto de la biblioteca de Universitaria de Cornell, y puede ser consultada.

Catherine J. Allen (con agradecimiento a Bill Isbell, Mary Jo Dudley y Enrique Mayer por sus contribuciones)

\section{BIBLIOGRAFÍA DE BILLIE JEAN ISBELL}

Colección en la que se incluyen unas 1500 fotografías anotadas, trece canciones y una selección de sus obras.

(2005). The Billie Jean Isbell Andean Collection. Recuperado de http://isbellandes. library.cornell.edu/ Estas publicaciones traen los enlaces que se muestran a continuación).

\section{Libros}

(1985 [1978]). To Defend Ourselves: Ecology and Ritual in an Andean Village. Prospect Heights, IL: Waveland Press [primera edición University of Texas Press 1978] URL: http://hdl.handle.net/1813/2135

(2005 [1978]). Para defendernos: ecología y ritual en un pueblo andino. Centro de Estudios Regionales Andinos Bartolomé de las Casas.

(2009). Finding Cholita. University of Illinois Press. 
Artículos en libros y revistas

(1972). Acquisition of Quechua morphology: An application of the Berko Test. Papers in Andean Linguistics, 1, 79-129.

(1974) Parentesco andino y reciprocidad: kuyaq - los que nos aman. En G. Alberti y Mayer, E. (Eds.), Reciprocidad e intercambio en los Andes Peruanos (pp. 110-52). IEP.

(1976) La otra mitad esencial: un estudio de complementariedad sexual en los Andes. Estudios Andinos, 5 (12), 37-56.

(1977). «Those Who Love Me»: an analysis of Andean kinship and reciprocity within a ritual context. En R. Bolton yMayer, E. (Eds)Andean Kinship and Marriage (pp.81-105).. American Anthropological Association,.

(1982). Culture Confronts Nature in the Dialectical World of the Tropics. En A. F. Aveni y Urton, G(Eds..),Ethno-AstronomyandArchaeo-Astronomyinthe American Tropics (pp. 353-363). New York: Annals of the New York Academy of Sciences, vol. $385 .$. URL: http://hdl.handle.net/1813/2193

(1994). Shining Path and peasant responses in rural Ayacucho. En D. S. Palmer (Ed.). Shining Paths of Peru (pp. 59-82).: St Martin's Press

(1995). Women's Voices: Lima 1975. En D. Tedlock y Mannheim, B. (Eds. The Dialogic Emergence of Culture (pp. 54-74). University of Illinois Press.

(1997). De inmaduro a duro: Lo simbólico femenino y los esquemas andinos de género. En D. Arnold (Ed.), Más allá del silencio: las fronteras de género en los Andes (pp. 253-301). Instituto de Lengua y Cultura Aymara. URL: http://hdl.handle. net/1813/2474

(1998). Violence in Peru: Performances and dialogues. American Anthropologist, 100, 283-292. URL: http://hdl.handle.net/1813/2195

(2003). You Can Make a Difference: Human Rights as the Subject Matter for a FirstYear Writing Seminar. En J. Monroe (ed.), Local Knowledges, Local Practices: Writing in the Disciplines at Cornell (pp. 90-98). University of Pittsburgh Press.

(2004). Protest arts from Ayacucho, Peru: Song and visual artworks as validation of experience. En G. Delgado y Schechter, J. M. (Eds.), Quechua expresivo: la inscripción de voces andinas / Quechua verbal artistry: the inscription of Andean voices (pp. 237-262). Bonn Americanist Studies.

(2009a). Written on my body. En P. Ghassem-Fachandi (Ed.), Violence: Ethnographic Encounters (pp. 15-34). : Berg.

(2009b). Lessons from Vicos. Anthropology in Action, 16, 41-54. 
(2010). El retorno de Cornell a Vicos, 2005. En R. Bolton, Greaves, T. y Zapata, F. (Eds.), 50 Años de Antropología Aplicada en el Perú. Vicos y otras experiencias (pp. 371-410). IEP.

(2011). Cornell returns to Vicos, 2005. En T.C. Greaves, Bolton, R. y Zapata, F. (Eds..), Vicos and Beyond: a Half Century of Applying Anthropology in Peru (p. 283-308). AltaMira Press.

Sin fecha. Public Secrets from Peru. A play [obra de teatro, manuscrito compuesto en 2003] URL: http://hdl.handle.net/1813/2196

\section{Publicaciones en coautoría}

Isbell, B. J. y Roncalla-Fernández, F. A. (1977). The ontogenesis of metaphor: Riddle games among Quechua speakers seen as cognitive discovery procedures. Journal of Latin American Lore, 3, 19-49. URL: http://hdl.handle.net/1813/2271

Franquemont,Edward M.,Franquemont, Christiney Isbell, BillieJean(1992).Awaqñawin: elojodel tejedor, lapráctica de la cultura en eltejido. RevistaAndina, 1, año 10,47-80. URL: http://hdl.handle.net/1813/2270 